\title{
BMJ Open Manual ventilation to prevent hypoxaemia during endotracheal intubation of critically ill adults: protocol and statistical analysis plan for a multicentre randomised trial
}

Jonathan D Casey, ${ }^{1}$ David R Janz, ${ }^{2}$ Derek W Russell, ${ }^{3}$ Derek J Vonderhaar, ${ }^{2}$ Aaron M Joffe, ${ }^{4}$ Kevin M Dischert, ${ }^{4}$ Ryan M Brown, ${ }^{1}$ Michael G Lester, ${ }^{1}$ Aline N Zouk, ${ }^{3}$ Swati Gulati, ${ }^{3}$ William S Stigler, ${ }^{3}$ Todd W Rice, ${ }^{1}$ Matthew W Semler, ${ }^{1}$ the PreVent Investigators and the Pragmatic Critical Care Research Group

To cite: Casey JD, Janz DR, Russell DW, et al. Manual ventilation to prevent hypoxaemia during endotracheal intubation of critically ill adults: protocol and statistical analysis plan for a multicentre randomised trial. BMJ Open 2018;8:e022139. doi:10.1136/ bmjopen-2018-022139

- Prepublication history and additional material for this paper are available online. To view these files, please visit the journal online (http://dx.doi. org/10.1136/bmjopen-2018022139).

Received 5 February 2018 Revised 8 June 2018 Accepted 17 July 2018

\section{Check for updates}

(c) Author(s) (or their employer(s)) 2018. Re-use permitted under CC BY-NC. No commercial re-use. See rights and permissions. Published by BMJ.

For numbered affiliations see end of article.

\section{Correspondence to} Dr Jonathan D Casey; Jonathan.d.Casey@vanderbilt. edu

\section{ABSTRACT}

Introduction Hypoxaemia is the most common complication during endotracheal intubation of critically ill adults, and it increases the risk of cardiac arrest and death. Manual ventilation between induction and intubation has been hypothesised to decrease the incidence of hypoxaemia, but efficacy and safety data are lacking. Methods and analysis The Preventing Hypoxemia with Manual Ventilation during Endotracheal Intubation trial is a prospective, multicentre, non-blinded randomised clinical trial being conducted in seven intensive care units in the USA. A total of 400 critically ill adults undergoing endotracheal intubation will be randomised $1: 1$ to receive prophylactic manual ventilation between induction and endotracheal intubation using a bag-valve-mask device or no prophylactic ventilation. The primary outcome is the lowest arterial oxygen saturation between induction and 2 min after successful endotracheal intubation, which will be analysed as an unadjusted, intention-totreat comparison of patients randomised to prophylactic ventilation versus patients randomised to no prophylactic ventilation. The secondary outcome is the incidence of severe hypoxaemia, defined as any arterial oxygen saturation of less than $80 \%$ between induction and $2 \mathrm{~min}$ after endotracheal intubation. Enrolment began on 2 February 2017 and is expected to be complete in May 2018.

Ethics and dissemination The trial was approved by the institutional review boards or designees of all participating centres. The results will be submitted for publication in a peer-reviewed journal and presented at one or more scientific conferences.

Trial registration number NCT03026322; Pre-results.

\section{INTRODUCTION}

Endotracheal intubation is common in the care of critically ill patients and is frequently associated with complications. ${ }^{1-3}$ Hypoxaemia occurs in approximately $40 \%$ of intubations outside the operating room, and is associated

\section{Strengths and limitations of this study}

- This ongoing pragmatic trial will provide the first comparison of clinical outcomes with prophylactic ventilation versus no prophylactic ventilation during endotracheal intubation of critically ill adults.

- Enrolling patients at multiple centres using broad inclusion criteria will enhance the generalisability of the findings.

- The nature of the study intervention does not allow blinding.

- Despite being one of the largest randomised trials to examine endotracheal intubation of critically ill patients, statistical power will be inadequate to detect differences between study groups in uncommon outcomes (eg, operator-reported aspiration).

with an increased risk for cardiac arrest and death. $^{24-7}$

Rapid sequence intubation is the nearly simultaneous administration of a sedative and neuromuscular blocking agent (paralytic) to facilitate endotracheal intubation. This technique is intended to maximise the chances of intubation on the first laryngoscopy attempt and minimise the risk of aspiration. Rapid sequence intubation has been shown to increase the incidence of successful intubation on the first laryngoscopy attempt and to decrease complications compared with intubation without neuromuscular blockade. ${ }^{8-10}$ Regardless of the choice of induction agent and neuromuscular blocker, rapid sequence intubation involves an inherent delay between medication administration and onset of paralysis, at which time laryngoscopy is initiated. The relative benefits and risks of providing ventilation to patients during this interval are 


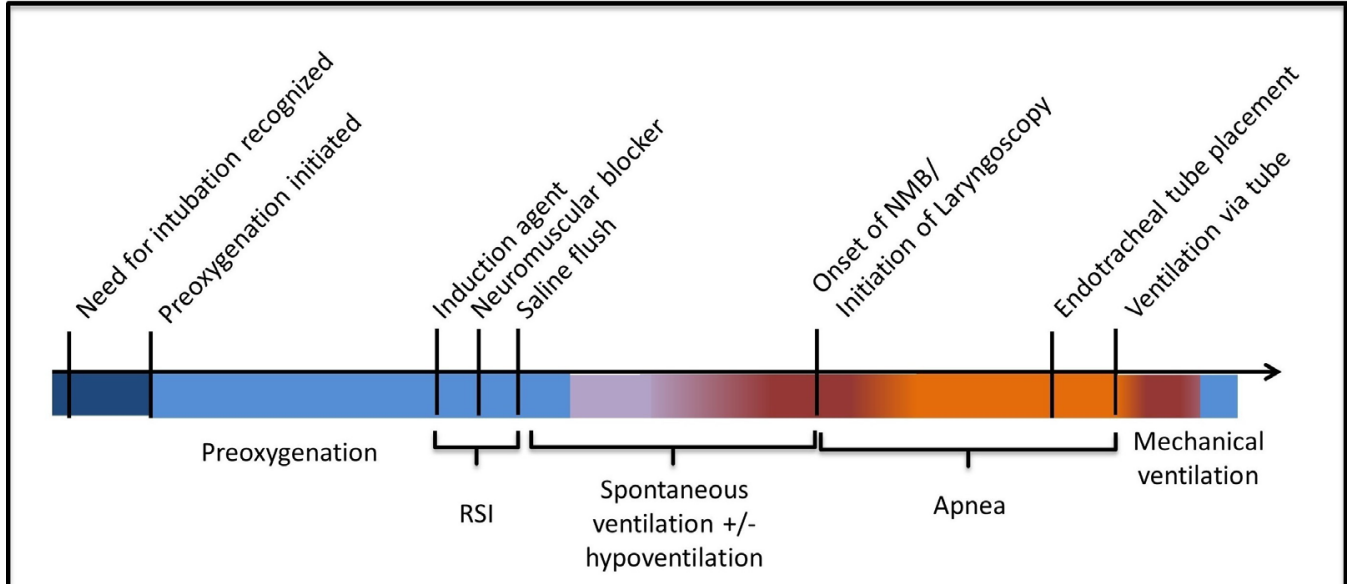

Figure 1 Phases of rapid sequence intubation without prophylactic manual ventilation. NMB, neuromuscular blockade; RSI, rapid sequence intubation.

unknown. Some airway management texts and guidelines recommend that, for patients who are not hypoxaemic, no ventilation be provided between induction and intubation, allowing the patient to remain hypopnoeic or apnoeic with the onset of sedation and neuromuscular blockade (figure 1). ${ }^{11-18}$ This approach prioritises minimising the potential risk of aspiration over any potential benefit of preventing the development of hypoxaemia and hypercapnia. Other airway management texts and guidelines recommend the provision of manual ventilation between induction and intubation using a bag-valvemask device for all patients, including those who are not hypoxaemic (referred to hereafter as 'prophylactic ventilation') (figure 2). ${ }^{1} 1717$ 19-22 This approach prioritises the potential benefit of preventing the development of hypoxaemia and hypercapnia over the potential risk of aspiration. National and international surveys of anaesthesiologists demonstrate that up to $50 \%$ of anaesthesia practitioners report routinely performing prophylactic ventilation between induction and intubation during out-of-operating room intubations. ${ }^{23}{ }^{24}$ The most recent published guidelines on intubation of critically ill adults recognises the arguments for and against prophylactic ventilation without making any recommendation as to whether or not it should be used..$^{25}$

Hundreds of thousands of critically ill adults require endotracheal intubation each year in the USA alone, but despite the frequency of this procedure, there are currently no high-quality data available to help providers understand the potential benefits and risks of providing prophylactic ventilation between induction and intubation. ${ }^{26}$ To address this knowledge gap, we designed a multicentre, randomised trial comparing prophylactic ventilation to no prophylactic ventilation during endotracheal intubation of critically ill adults. We hypothesise that, compared with no prophylactic ventilation, prophylactic ventilation will significantly increase the lowest arterial oxygen saturation between induction and $2 \mathrm{~min}$ after endotracheal intubation.

\section{METHODS AND ANALYSIS}

This manuscript was written in accordance with Standard Protocol Items: Recommendations for Interventional

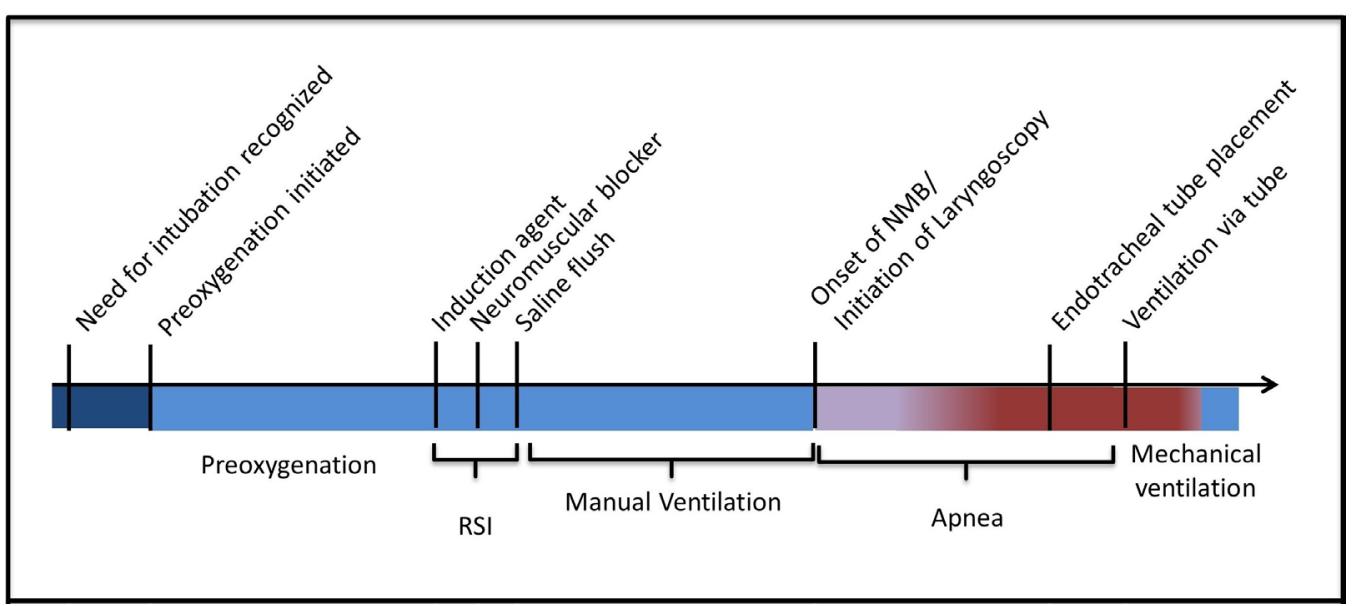

Figure 2 Phases of rapid sequence intubation with prophylactic manual ventilation. NMB, neuromuscular blockade; RSI, rapid sequence intubation. 


\begin{tabular}{|c|c|c|c|c|c|c|c|}
\hline & \multicolumn{7}{|c|}{ STUDY PERIOD } \\
\hline & \multirow{2}{*}{$\begin{array}{c}\text { Enrolment } \\
\text { Decision to } \\
\text { perform } \\
\text { ETI }\end{array}$} & \multirow{2}{*}{$\begin{array}{c}\text { Allocation } \\
\text { Prior to } \\
\text { Induction }\end{array}$} & \multicolumn{4}{|c|}{ On-Study } & \multirow{2}{*}{$\begin{array}{c}\text { Follow Up } \\
\text { Discharge or } 30 \\
\text { days after } \\
\text { enrollment }\end{array}$} \\
\hline TIMEPOINT & & & $\begin{array}{l}\text { Induction } \\
\text { \& NMB }\end{array}$ & ETI & $\begin{array}{c}2 \text { min } \\
\text { post-ETI }\end{array}$ & $\begin{array}{l}48 \mathrm{hrs} \\
\text { post-ETI }\end{array}$ & \\
\hline ENROLMENT: & $\bar{x}$ & & & & & & \\
\hline Eligibility screen & $\mathrm{X}$ & & & & & & \\
\hline Allocation & & $x$ & & & & & \\
\hline \multicolumn{8}{|l|}{ INTERVENTIONS: } \\
\hline \multicolumn{8}{|l|}{$\begin{array}{r}\text { Prophylactic } \\
\text { Ventilation }\end{array}$} \\
\hline $\begin{array}{r}\text { Screening for } \\
\text { contraindications }\end{array}$ & $X$ & $\mathrm{X}$ & $X$ & $X$ & & & \\
\hline \multicolumn{8}{|l|}{$\begin{array}{r}\text { No Prophylactic } \\
\text { Ventilation }\end{array}$} \\
\hline $\begin{array}{r}\text { Screening for } \\
\text { contraindications }\end{array}$ & $\mathrm{X}$ & $\mathrm{X}$ & $\mathrm{X}$ & $x$ & & & \\
\hline \multicolumn{8}{|l|}{ ASSESSMENTS: } \\
\hline $\begin{array}{r}\text { Baseline } \\
\text { Variables }\end{array}$ & $\mathrm{X}$ & $\mathrm{x}$ & & & & & \\
\hline \multirow{2}{*}{$\begin{array}{r}\text { Peri-procedural } \\
\text { variables } \\
\text { Clinical Outcomes }\end{array}$} & & $\mathrm{x}$ & $\mathrm{x}$ & $X$ & $\mathrm{X}$ & & \\
\hline & & & & & & $\mathrm{X}$ & $X$ \\
\hline
\end{tabular}

Figure 3 Standard Protocol Items: Recommendations for Interventional Trials (SPIRIT) checklist. Enrolment, interventions and assessments. Baseline variables obtained from electronic medical record include: demographic characteristics, indication for intubation, history of pulmonary disease, severity of illness at enrolment, risk factors for aspiration, non-invasive ventilator use and highest fraction of inspired oxygen in the 6 hours prior to intubation. Periprocedural variables, including oxygen saturation at induction, lowest arterial oxygen saturation between induction and 2 min following endotracheal intubation and time to intubation will be collected by a trained, independent observer, not affiliated with the performance of the procedure. Clinical outcomes include: vital status, number of ventilator-free days to 28 days, and number of intensive care unit-free days to 28 days. ETI, endotracheal intubation; NMB, neuromuscular blockade.

Trials (SPIRIT) guidelines (figure 3; SPIRIT checklist in online supplementary file 1 , section 1$).{ }^{27}$

\section{Study design}

The Preventing Hypoxemia with Manual Ventilation during Endotracheal Intubation (PreVent) trial is a multicentre, parallel-group, unblinded, pragmatic randomised trial being conducted in seven intensive care units (ICUs) at five medical centres across the USA. The trial compares prophylactic manual ventilation between induction and endotracheal intubation using a bag-valve-mask device to no prophylactic ventilation during endotracheal intubation of critically ill adults. Enrolment began on 2 February 2017 and is expected to be complete in May 2018. The primary outcome is lowest arterial oxygen saturation between induction and 2 min after endotracheal intubation. The trial was registered with ClinicalTrials.gov prior to initiation of patient enrolment (ClinicalTrials. gov identifiers: NCT03026322). An independent data and safety monitoring board (DSMB) is monitoring the progress and safety of the trial.

\section{Patient and public involvement}

Patients and the public were not involved in identifying the research question or the design of the study. We plan to disseminate the results of the study to the public at the completion of the trial.

\section{Study sites}

The trial is being conducted at seven academic ICUs across the USA: a 35-bed medical ICU at Vanderbilt University Medical Centre in Nashville, Tennessee; a 38-bed medical, cardiac and neurological ICU at University Medical Centre in New Orleans, Louisiana; a 33-bed medical ICU at Ochsner Medical Centre in New Orleans, Louisiana; a 25-bed medical ICU at University of Alabama 
at Birmingham Medical Centre in Birmingham, Alabama; and a 17-bed medical ICU, a 30-bed neurological ICU and 24-bed trauma ICU at University of Washington Harborview Medical Centre in Seattle, Washington.

\section{Population}

The inclusion criteria for the trial are:

1. Adult patient (age $\geq 18$ years);

2. Located in a participating ICU;

3. Treating clinicians have determined endotracheal intubation is required;

4. Planned procedural approach includes administration of an induction agent (with or without neuromuscular blockade); and

5. First operator who routinely performs endotracheal intubation in the participating ICU.

The exclusion criteria for the trial are:

1. Pregnant women;

2. Prisoners;

3. Patients for whom the treating clinicians feel the urgency of the intubation precludes safe performance of study procedures; and

4. Patients for whom a treating clinician feels a specific approach to ventilation between induction and intubation is required.

Patients are not excluded based on oxygen saturation at enrolment. A patient flow chart diagram describing the number of patients screened for the trial, the number excluded and the reasons for exclusion will be included in the manuscript reporting the results of the trial.

\section{Randomisation and treatment allocation}

Enrolled patients are randomised in a 1:1 ratio to prophylactic ventilation or no prophylactic ventilation. The allocation sequence was generated by study personnel at the coordinating centre using computerised randomisation in permuted blocks, stratified by study ICU. Study group assignments were placed in sequentially numbered opaque envelopes and distributed to the study ICUs. Group assignment remains concealed from local study personnel and treating clinicians until the determination has been made that a patient (1) requires endotracheal intubation, (2) meets all inclusion criteria and (3) meets no exclusion criteria-at which point the enveloped is opened. After enrolment and randomisation, patients, treating clinicians and study personnel at the local site are not blinded to study group assignment.

\section{Study interventions}

\section{Definitions}

Ventilation between induction and endotracheal intubation refers to the delivery of positive pressure breaths using a non-invasive ventilator or a bag-valve-mask device. Prophylactic ventilation describes ventilation administered to a patient without hypoxaemia to prevent the development of hypoxaemia. Separately, ventilation may represent treatment of hypoxaemia for patients who are experiencing hypoxaemia at the initiation of ventilation.
The focus of this trial is on the administration of manual ventilation with a bag-valve-mask device to prevent the development of hypoxaemia. Treatment of hypoxaemia with manual ventilation is not considered prophylactic ventilation and is allowed at any time in either study group. Administration of ventilation with a non-invasive ventilator between induction and laryngoscopy is prohibited in both study groups because it represents a source of confounding with regard to the provision of prophylactic ventilation. Preoxygenation prior to induction is allowed in either group with any preoxygenation modality, including non-invasive ventilation.

\section{Prophylactic ventilation}

For patients assigned to the prophylactic ventilation group, manual ventilation is provided using a bag-valvemask device beginning at induction and continuing until the initiation of laryngoscopy. If more than one attempt at laryngoscopy occurs, manual ventilation using a bag-valvemask device may be reinstituted between laryngoscopy attempts. Manual ventilation may be discontinued at any point if felt by the treating clinicians to be necessary for patient safety.

Manual ventilation with a bag-valve-mask device is a routinely employed technique familiar to clinicians who perform endotracheal intubation in the ICU. In keeping with the pragmatic nature of the trial, manual ventilation with a bag-valve-mask device is provided during the trial by the same treating clinicians who would perform the intervention outside of a research setting. Trainees responsible for airway management in participating units received an educational intervention prior to the beginning of enrolment reviewing best practices in manual ventilation using a bag-valve-mask device. This training emphasised proper mask placement, airway patency manoeuvres, positive end-expiratory pressure (PEEP), oxygen flow rates and ideal ventilation rates and volumes. In addition, the group assignment sheet for the prophylactic ventilation group includes reminders of best practices for manual ventilation using a bag-valve-mask device, including instructions to use: oxygen flow rates of at least $15 \mathrm{~L}$ per minute; a PEEP valve set to $5-10 \mathrm{~cm}$ of water; an oral airway; a two-handed mask seal performed by the intubating clinician with a head tilt-chin lift (with a stock photograph demonstrating proper technique); and ventilation at 10 breaths per minute until laryngoscopy. Details of patients' receipt of manual ventilation between induction and intubation are prospectively recorded. Failure to administer manual ventilation with a bag-valvemask device beginning at induction is documented as a protocol violation.

\section{No prophylactic ventilation}

Patients assigned to the no prophylactic ventilation group do not receive prophylactic ventilation between induction and intubation. Manual ventilation is allowed as treatment (1) for hypoxaemia (oxygen saturation <90\%) or (2) following a failed laryngoscopy attempt. In addition, 
manual ventilation may be initiated at any point if felt by the treating clinicians to be necessary for the safe treatment of the patient. Details of patients' receipt of ventilation between induction and endotracheal intubation are prospectively recorded. Administration of ventilation using a bag-valve-mask device before the first attempt at laryngoscopy in a patient who does not experience hypoxaemia (oxygen saturation $<90 \%$ ) is documented as a protocol violation. The group assignment sheet for the no prophylactic ventilation group includes reminders that apnoeic oxygenation is allowed, that non-invasive ventilation should be removed at induction and that bag-valvemask ventilation is allowed for oxygen saturation $<90 \%$.

\section{Cointerventions}

Study group assignment determines only the approach to prophylactic ventilation between induction and endotracheal intubation. Treating clinicians determine the need for intubation, approach to preoxygenation, patient positioning, choice and timing of medications for induction and neuromuscular blockade, choice of laryngoscope type and size, use of cricoid pressure and use of additional airway management equipment.

\section{Data collection}

A trained, independent observer not affiliated with the performance of the procedure collects data for key periprocedural outcomes, including oxygen saturation and systolic blood pressure at induction, lowest arterial oxygen saturation and systolic blood pressure between induction and $2 \mathrm{~min}$ following intubation, vasopressor administration and time to intubation. The accuracy of data collection by the independent observers is confirmed by concurrent assessment of the same outcomes by the primary investigators for a convenience sample of approximately $10 \%$ of study intubations.

Cormack-Lehane grade of glottic view, ${ }^{28}$ subjective difficulty of intubation and airway complications during the procedure are reported by the operator. Operators self-report their prior intubating experience at the time of each study intubation.

Study personnel collect data on baseline characteristics, prelaryngoscopy and postlaryngoscopy management, and clinical outcomes from the medical record. The following variables are collected:

1. Baseline. Age, gender, height, weight, body mass index, race, Acute Physiology and Chronic Health Evaluation (APACHE II score), active medical problems at the time of intubation, active comorbidities complicating intubation, comorbidities known to increase risk of aspiration (history of gastro-oesophageal reflux, narcotic use, functional or mechanical gastrointestinal obstruction, previous oesophageal surgery, head injury, active emesis, or active upper gastrointestinal bleeding), indication for intubation, reintubation status, preoxygenation technique, operator experience, non-invasive ventilator use, vasopressor use, arterial blood gas results, and the highest fraction of inspired oxygen delivered $\left(\mathrm{FiO}_{2}\right)$, lowest systolic blood pressure observed and lowest oxygen saturation observed in the 6 hours preceding intubation.

2. Periprocedural. Preprocedural fluid and vasopressors. Date and time of sedative administration, saturation at time of sedative administration, type and dose of sedative, type and dose of neuromuscular blocker, use of manual ventilation starting at the time of induction, any use of ventilation during the intubation, indication for ventilation (study assignment, oxygen saturation less than $90 \%$, following a failed attempt, other), use of oral or nasal airway, use of cricoid pressure, laryngoscope type and size, total number of attempts, airway grade, airway difficulty, use of rescue device(s), need for additional operators, date and time of first laryngoscopy attempt, date and time of successful intubation, mechanical complications (oesophageal intubation, airway trauma), bradycardia and the presence of aspiration between induction and intubation (reported by operator).

3. 0-48 hours. All chest imaging obtained within the first 48 hours after intubation, postintubation shock or cardiac arrest, highest and lowest $\mathrm{SaO}_{2}, \mathrm{FiO}_{2}$, PEEP and systolic blood pressure in the 1, 6 and 24 hours after intubation.

4. In-hospital outcomes. Ventilator-free days, ICU-free days and in-hospital mortality. Definitions for ventilator-free days and ICU-free days can be found in the online supplementary file 1 , sections 2 and 3 .

\section{Primary outcome}

The primary outcome is the lowest arterial oxygen saturation measured by continuous pulse oximetry $\left(\mathrm{SpO}_{2}\right)$ between induction and $2 \mathrm{~min}$ after endotracheal intubation ('lowest arterial oxygen saturation') as documented by the independent observer.

\section{Secondary outcome}

The single, prespecified, secondary outcome is the incidence of severe hypoxaemia, defined as any oxygen saturation less than $80 \%$ between induction and $2 \mathrm{~min}$ after endotracheal intubation. The optimal outcome for clinical trials attempting to improve oxygenation during endotracheal intubation of critically ill adults is unknown. In addition to the primary outcome of lowest arterial oxygen saturation as a continuous variable, some experts have recommended examination of the endpoint of 'severe hypoxemia' as a dichotomous outcome. We therefore highlight the incidence of oxygen saturation less than $80 \%$ as our prespecified approach to analysis of lowest oxygen saturation as a dichotomous outcome. All additional outcomes are exploratory and will be considered hypothesis generating.

\section{Main safety outcomes}

The main safety outcomes will be the lowest $\mathrm{SpO}_{2}$, highest $\mathrm{FiO}_{2}$ and highest PEEP in the time period of 6 to 24 hours postintubation. The outcomes of $\mathrm{SpO}_{2}, \mathrm{FiO}_{2}$ and PEEP 
are selected to capture objective clinical manifestations of periprocedural aspiration. The time point of 6 to 24 hours postintubation is chosen to account for the practice, at some centres, of initiating patients at $100 \% \mathrm{FiO}_{2}$ and low PEEP immediately after intubation, and subsequently titrating $\mathrm{FiO}_{2}$ and PEEP over several hours to achieve the target $\mathrm{SpO}_{2}$.

\section{Exploratory procedural outcomes}

- Cormack-Lehane grade of glottic view.

- Operator-assessed difficulty of intubation.

- Incidence of successful intubation on the first laryngoscopy attempt.

- Number of laryngoscopy attempts.

- Time from induction to successful intubation.

- Incidence of oesophageal intubation.

- Need for additional airway equipment or a second operator.

- Incidence of lowest oxygen saturation less than $90 \%$.

- Change in oxygen saturation from induction to lowest oxygen saturation.

- Incidence of desaturation, defined as a change in oxygen saturation of more than $3 \%$ from induction to 2 min after endotracheal intubation.

\section{Exploratory safety outcomes}

- Operator-reported aspiration during the procedure, defined as visualisation of oropharyngeal or gastric contents in the pharynx, larynx or trachea between induction and completion of airway management.

- New infiltrate on chest X-ray in the 48 hours following intubation, as determined by an independent reviewer; details in online supplementary file 1 , section 4 .

- New pneumothorax within 24 hours of intubation, as determined by an independent reviewer; details in online supplementary file 1 , section 4 .

- New pneumomediastinum within 24 hours of intubation, as determined by an independent reviewer; details in online supplementary file 1 , section 4 .

- Lowest systolic blood between induction and $2 \mathrm{~min}$ after endotracheal intubation.

- New systolic blood pressure $<65 \mathrm{~mm} \mathrm{Hg}$ or new vasopressor administration between induction and $2 \mathrm{~min}$ after endotracheal intubation.

- Cardiac arrest within 1 hour of intubation.

- Death within 1 hour of intubation.

- Lowest $\mathrm{SpO}_{2}$, highest $\mathrm{FiO}_{2}$ and highest PEEP from 0 to 1 and $1-6$ hours.

- The composite of operator-reported pulmonary aspiration, new chest X-ray infiltrate, OR lowest oxygen saturation $<80 \%$ between induction and completion of endotracheal intubation.

\section{Exploratory clinical outcomes}

- Ventilator-free days to 28 days.

- ICU-free days to 28 days.

- In-hospital mortality.

\section{Sample size estimation}

Full details of the initial sample size calculation can be found in the online supplementary file 1 , section 5 . In short, using PS V.3.1.29 and assuming an SD of $14 \%$ in lowest oxygen saturation (the primary outcome) and less than 5\% missing data, we calculated that enrolling 350 patients would provide $90 \%$ power to detect a difference of $5 \%$ between groups in lowest oxygen saturation at a two-sided alpha of 0.05 . The trial protocol and DSMB charter specified that the DSMB would recommend sample size re-estimation at the interim analysis if the SD for lowest oxygen saturation in the control arm was larger than $14 \%$, in order to prevent the final study from being underpowered to detect the planned difference between groups in lowest oxygen saturation. At the interim analysis, the observed SD for lowest oxygen saturation in the control arm was $15 \%$. To maintain $90 \%$ statistical power to detect a $5 \%$ difference between groups in lowest oxygen saturation, the DMSB recommended increasing the sample size to 400 patients. Additional details of the sample size re-estimation can be found in the online supplementary file 1 , section 6 .

\section{DSMB and interim analysis}

An independent DSMB was appointed to oversee the conduct of the trial and review one interim analysis (DSMB charter available in the online supplementary file 1 , section 7). The DSMB was composed of two academic intensivists experienced in the conduct of clinical trials. The DSMB conducted a single interim analysis for efficacy and safety at the anticipated halfway point of the trial, after enrolment of 175 patients. The stopping boundary for efficacy was specified as a $p$ value of 0.001 or less for the difference between groups in the primary outcome. Use of a conservative Haybittle-Peto boundary $(\mathrm{p}<0.001)$ allows the final analysis to be performed using an unchanged level of significance $(\mathrm{p}=0.05)$. The primary determination of safety was based on the highest $\mathrm{FiO}_{2}$ and highest PEEP between 6 and 24 hours after intubation. If (1) the $p$ value for the difference between study groups in both of these physiologic variables was less than 0.001, (2) the difference between groups in both physiological variables was concordant in direction with the point estimate for in-hospital mortality, and (3) the p value for the difference between study groups in in-hospital mortality was less than 0.1 , it was recommended that the study be stopped early for safety.

The DSMB was also provided with data in each group on the rates of operator-reported aspiration and new infiltrates, pneumothorax or pneumomediastinum on chest imaging. Although no prespecified rules dictated stopping based on operator-reported aspiration or imaging findings without associated changes in physiological or clinical outcomes, the DSMB reserved the right to stop the trial at any point, request additional data or interim analyses or request modifications of the study protocol as required to protect patient safety. 
At the time of submission of this manuscript, the DSMB has completed the sole planned interim analysis following the enrolment of the first 175 patients. The DSMB has recommended continuing the trial to completion with the only change being to increase the sample size to 400 patients, as described above.

Additional details on data storage, patient privacy and the prespecified process for protocol changes can be found in the online supplementary file 1, sections 8 and 9.

\section{Statistical analysis principles}

All analyses will be performed using Stata V.15.1 (StataCorp. 2017. Stata Statistical Software: Release 15. College Station, Texasm USA: StataCorp LLC) and confirmed with SPSS V.25 (IBM Corp. Released 2017. IBM SPSS Statistics for Windows, V.25.0. Armonk, New York, USA: IBM Corp) or R V.3.2.0 (R Foundation for Statistical Computing, Vienna, Austria).

Continuous variables will be reported as mean \pm SD or median and IQR; categorical variables will be reported as frequencies and proportions. Between-group comparisons will be made with the Mann-Whitney rank-sum test for continuous variables, and the $\chi^{2}$ test or Fishers exact test for categorical variables. Agreement between continuous variables measured independently by two observers will be examined using Spearman rank correlation coefficient and Bland-Altman analysis. A two-sided $\mathrm{p}$ value $<0.05$ will indicate statistical significance.

\section{Primary analysis}

The primary analysis will be an unadjusted, intention-totreat comparison of patients randomised to prophylactic ventilation versus patients randomised to no prophylactic ventilation with regard to the primary outcome of lowest arterial oxygen saturation between induction and $2 \mathrm{~min}$ after endotracheal intubation. The difference between the two study groups will be compared using the MannWhitney rank-sum test.

\section{Secondary analyses}

We will conduct the following prespecified secondary analyses:

\section{Secondary and exploratory outcomes}

We will perform unadjusted, intention-to-treat analyses comparing patients in the prophylactic ventilation group to the no prophylactic ventilation group with regard to each of the prespecified secondary and exploratory outcomes. Continuous outcomes will be compared with the Mann-Whitney rank-sum test and categorical variables with the $\chi^{2}$ test.

\section{Per-protocol analysis}

We will perform a per-protocol analysis comparing patients who received prophylactic manual ventilation beginning at induction (regardless of group assignment) to patients who did not receive prophylactic manual ventilation beginning at induction (regardless of group assignment). Patients who were hypoxaemic at induction and received manual ventilation as treatment for hypoxaemia will be analysed in the group to which they were assigned.

\section{Effect modification (subgroup analyses)}

We will examine whether prespecified baseline variables modify the effect of study group on the primary outcome. We will evaluate for effect modification by fitting a linear regression model for the primary outcome of lowest arterial oxygen saturation. Independent variables will include study group assignment, the potential effect modifier variable of interest and the interaction between the two (eg, study group*oxygen saturation at induction). Significance will be determined by the $\mathrm{p}$ value for the interaction term, with values less than 0.10 considered suggestive of a potential interaction and values less than 0.05 considered to confirm an interaction. Subgroups derived from categorical variables will be displayed as a forest plot. Continuous variables will be analysed using restricted cubic splines with 3-5 knots and preferentially displayed as continuous variables using a locally weighted regression or partial effects plots. If the presentation of data requires it, dichotomisation of continuous variables for inclusion in a forest plot will be performed. Prespecified subgroups that may modify the effect of prophylactic ventilation include:

1. Predicted lowest arterial oxygen saturation ('risk of hypoxemia') as calculated by a prespecified multivariable model (continuous variable).

2. Oxygen saturation at induction (continuous variable).

3. Highest $\mathrm{FiO}_{2}$ received in the 6 hours prior to intubation (continuous variable).

4. Receipt of non-invasive ventilation in the 6 hours prior to intubation (yes/no).

5. Indication for intubation (hypoxaemic respiratory failure, not hypoxaemic respiratory failure).

6. Neuromuscular blocking agent (depolarising, non-depolarising, none).

7. APACHE II score at enrolment (continuous variable).

8. Body mass index (continuous variable).

9. Operator's prior number of endotracheal intubations (continuous variable).

10. Operator training (pulmonary/critical care medicine, anaesthesia).

11. Type of laryngoscope (direct laryngoscope, video laryngoscope).

\section{Multivariable modelling to account for confounding}

To account for relevant confounders, we will develop a linear regression model with the primary outcome as the dependent variable and study group and relevant confounders included as independent variables (age, APACHE II score at enrolment, oxygen saturation at induction, highest $\mathrm{FiO}_{2}$ delivered in the 6 hours prior to intubation and receipt of non-invasive ventilation in the 6 hours prior to intubation). 


\section{Missing data}

Based on prior trials in similar settings, we anticipate less than $5 \%$ missing data for the primary outcome. For the primary analysis, missing data will not be imputed. As sensitivity analyses, the primary analysis will be repeated with missing data imputed by (1) assigning a value of ' 0 ' to data missing for the lowest arterial oxygen saturation in the prophylactic ventilation group and ' 100 ' to data missing for the lowest arterial oxygen saturation in the no prophylactic ventilation group, and (2) assigning a value of ' 100 ' to data missing for the lowest arterial oxygen saturation in the prophylactic ventilation group and a value of ' 0 ' to data missing from the no prophylactic ventilation group.

\section{Corrections for multiple testing}

We have prespecified a single primary analysis of a single primary outcome. All additional analyses will be considered hypothesis generating, and no corrections for multiple comparisons will be performed.

\section{Trial status}

PreVent is an ongoing pragmatic trial comparing prophylactic ventilation using a bag-valve-mask to no prophylactic ventilation during endotracheal intubation of critically ill adults. Patient enrolment began on 2 February 2017, and we estimate that enrolment will end in May 2018.

\section{Ethics and dissemination Consent}

Prophylactic manual ventilation between induction and endotracheal intubation using a bag-valve-mask device and no prophylactic ventilation are each recommended approaches to endotracheal intubation of acutely ill adults. ${ }^{1325}$ Currently, no randomised trials or evidencebased guidelines support the choice of one approach over the other. Both approaches are used intermittently in current care in the study ICUs. Moreover, the current study specifically excludes patients for whom treating clinicians feel that the provision of prophylactic ventilation is either required or contraindicated.

The current study is felt by the investigators to represent minimal risk because the interventions studied (1) are used in current clinical care in the participating ICUs, (2) are interventions to which patients would be exposed even if not participating in research, (3) have no prior data to suggest the superiority of one approach over the other, and (4) are equivalent options from the perspective of the treating clinicians performing the procedure (otherwise the patient is excluded from the trial). Additionally, endotracheal intubation of critically ill adults is frequently a time-sensitive procedure for which obtaining informed consent is impractical. Given the minimal risk and impracticability of obtaining informed consent, a waiver of informed consent was requested from the Vanderbilt University Institutional Review Board.

\section{Institutional Review Board approval}

The trial was approved with waiver of informed consent (Institutional Review Board (IRB) 161962). All participating centres obtained local IRB approval (Louisiana State University Health Sciences Centre IRB Number 00000177 and Ochsner Clinic Foundation IRB Number 2017.119.B) or deferred to Vanderbilt University Medical Centre through a central IRB process (University of Alabama and University of Washington).

\section{Publication}

The results of the trial will be submitted for publication in a peer-reviewed journal and presented at one or more scientific conferences.

\section{CONCLUSION}

We describe, before the conclusion of enrolment or data unblinding, our approach to analysing the data from a pragmatic multicentre randomised trial comparing prophylactic ventilation between induction and intubation using a bag-valve-mask to no prophylactic ventilation (PreVent trial). We anticipate that this prespecified framework will enhance the utility of the reported result and allow readers to better judge the impact.

\section{Author affiliations}

${ }^{1}$ Division of Allergy, Pulmonary, and Critical Care Medicine, Vanderbilt University Medical Center, Nashville, Tennessee, USA

${ }^{2}$ School of Medicine, Section of Pulmonary/Critical Care \& Allergy/lmmunology, Louisiana State University, New Orleans, Louisiana, USA

${ }^{3}$ Division of Pulmonary, Allergy, \& Critical Care Medicine, University of Alabama at Birmingham, Birmingham, USA

${ }^{4}$ Department of Anesthesiology and Pain Medicinex, University of Washington, Seattle, Washington, USA

Contributors Study concept and design: JDC, MWS, DRJ and TWR; acquisition of data: JDC, DRJ, DWR, DJV, AMJ, KMD, RMB, MGL, ANZ, SG, WSS and MWS; analysis and interpretation of data, drafting of the manuscript and statistical analysis: JDC and MWS; critical revision of the manuscript for important intellectual content: JDC, RMB and MWS; study supervision: TWR.

Funding JDC was supported in part by the NIH (2T32HL087738-12). MWS was supported in part by the NHLBI (HL087738-09 and K12HL133117). DWR was supported in part by the NIH/NHLBI (T32HL105346-07). TWR was supported in part by the NIH (R34HL105869). Data collection used the Research Electronic Data Capture (REDCap) tool developed and maintained with Vanderbilt Institute for Clinical and Translational Research grant support (UL1 TR000445 from NCATS/ $\mathrm{NIH)}$.

Disclaimer The funding institutions had no role in (1) conception, design or conduct of the study, (2) collection, management, analysis, interpretation or presentation of the data or (3) preparation, review or approval of the manuscript.

Competing interests All authors completed and submitted the ICMJE Form for Disclosure of Potential Conflicts of Interest. The authors declared no potential conflicts of interest with the current work. TWR reported serving on an advisory board for Avisa Pharma, LLC, and as the Director of Medical Affairs for Cumberland Pharmaceuticals.

Patient consent Not required.

Ethics approval Institutional Review Board of Vanderbilt University Medical Center. Provenance and peer review Not commissioned; externally peer reviewed.

Data sharing statement This manuscript details the protocol and statistical analysis plan for an ongoing clinical trial. At present there are no data available for sharing. 
Open access This is an open access article distributed in accordance with the Creative Commons Attribution Non Commercial (CC BY-NC 4.0) license, which permits others to distribute, remix, adapt, build upon this work non-commercially, and license their derivative works on different terms, provided the original work is properly cited, appropriate credit is given, any changes made indicated, and the use is non-commercial. See: http://creativecommons.org/licenses/by-nc/4.0/.

\section{REFERENCES}

1. Schwartz DE, Matthay MA, Cohen NH. Death and other complications of emergency airway management in critically ill adults. A prospective investigation of 297 tracheal intubations. Anesthesiology 1995;82:367-76.

2. Simpson GD, Ross MJ, McKeown DW, et al. Tracheal intubation in the critically ill: a multi-centre national study of practice and complications. Br J Anaesth 2012;108:792-9.

3. Leibowitz $A B$. Tracheal intubation in the intensive care unit: extremely hazardous even in the best of hands. Crit Care Med 2006;34:2497-8.

4. Mort TC. The incidence and risk factors for cardiac arrest during emergency tracheal intubation: a justification for incorporating the ASA Guidelines in the remote location. $J$ Clin Anesth 2004;16:508-16.

5. Martin LD, Mhyre JM, Shanks AM, et al. 3,423 emergency tracheal intubations at a university hospital: airway outcomes and complications. Anesthesiology 2011;114:42-8.

6. Griesdale DE, Bosma TL, Kurth T, et al. Complications of endotracheal intubation in the critically ill. Intensive Care Med 2008;34:1835-42.

7. Semler MW, Janz DR, Lentz RJ, et al. Randomized Trial of Apneic Oxygenation during Endotracheal Intubation of the Critically III. Am J Respir Crit Care Med 2016;193:273-80.

8. Brown CA, Bair AE, Pallin DJ, et al. Techniques, success, and adverse events of emergency department adult intubations. Ann Emerg Med 2015;65:363-70.

9. Li J, Murphy-Lavoie H, Bugas C, et al. Complications of emergency intubation with and without paralysis. Am J Emerg Med 1999;17:141-3.

10. Jaber S, Amraoui J, Lefrant JY, et al. Clinical practice and risk factors for immediate complications of endotracheal intubation in the intensive care unit: a prospective, multiple-center study. Crit Care Med 2006;34:2355-61.

11. Wylie WD. The use of muscle relaxants at the induction of anaesthesia of patients with a full stomach. $\mathrm{Br} J$ Anaesth 1963;35:168-73.

12. Salem MR. Anesthetic management of patients with "a full stomach". A critical review. Anesth Analg 1970;49:47???55-55.
13. Walls RM. Rapid Sequence Intubation: In. Manual of Emergency Airway Management. Philadelphia: Lippincott Williams \& Wilkins, a Wolters Kluwer business, 2012:221-31.

14. Natanson $\mathrm{C}$, Shelhamer JH, Parrillo JE. Intubation of the trachea in the critical care setting. JAMA 1985;253:1160-5.

15. Caputo N, Azan B, Domingues R, et al. Emergency Department use of Apneic Oxygenation Versus Usual Care During Rapid Sequence Intubation: A Randomized Controlled Trial (The ENDAO Trial). Acad Emerg Med 2017;24:1387-94.

16. Stollings JL, Diedrich DA, Oyen LJ, et al. Rapid-sequence intubation: a review of the process and considerations when choosing medications. Ann Pharmacother 2014;48:62-76.

17. El-Orbany M, Connolly LA. Rapid sequence induction and intubation: current controversy. Anesth Analg 2010;110:1318-25.

18. Stept WJ, Safar P. Rapid induction-intubation for prevention of gastric-content aspiration. Anesth Analg 1970;49:633-6.

19. Nagelhout JJ. AANA journal course. Update for nurse anesthetists. Aspiration prophylaxis: is it time for changes in our practice? Aana $J$ 2003;71:299-303.

20. Herriger A, Frascarolo P, Spahn DR, et al. The effect of positive airway pressure during pre-oxygenation and induction of anaesthesia upon duration of non-hypoxic apnoea. Anaesthesia 2004;59:243-7.

21. Frerk C, Mitchell VS, McNarry AF, et al. Difficult Airway Society 2015 guidelines for management of unanticipated difficult intubation in adults. Br J Anaesth 2015;115:827-48.

22. Myatra SN, Ahmed SM, Kundra P, et al. The All India Difficult Airway Association 2016 guidelines for tracheal intubation in the Intensive Care Unit. Indian J Anaesth 2016;60:922-30.

23. Ehrenfeld JM, Cassedy EA, Forbes VE, et al. Modified rapid sequence induction and intubation: a survey of United States current practice. Anesth Analg 2012;115:95-101.

24. Schlesinger S, Blanchfield D. Modified rapid-sequence induction of anesthesia: a survey of current clinical practice. Aana $J$ 2001;69:291-8

25. Higgs A, McGrath BA, Goddard C, et al. Guidelines for the management of tracheal intubation in critically ill adults. $\mathrm{Br} J$ Anaesth 2018;120:323-52.

26. Pfuntner A, Wier LM, Stocks C. Most Frequent Procedures Performed in U.S. Hospitals, 2011: Statistical Brief \#165: In. Healthcare Cost and Utilization Project (HCUP) Statistical Briefs. Rockville (MD): Agency for Healthcare Research and Quality (US), 2013. http://www.ncbi.nlm.nih.gov/books/NBK174682/. (accessed 4 Jun 2018).

27. Chan AW, Tetzlaff JM, Gøtzsche PC, et al. SPIRIT 2013 explanation and elaboration: guidance for protocols of clinical trials. BMJ 2013;346:e7586 https://www.ncbi.nlm.nih.gov/pubmed/23303884.

28. Cormack RS, Lehane J. Difficult tracheal intubation in obstetrics. Anaesthesia 1984;39:1105-11.

29. Dupont WD, Plummer WD. Power and sample size calculations. A review and computer program. Control Clin Trials 1990;11:116-28. 